\title{
A meningocele complicating mastoidectomy - case considerations
}

\author{
Dan-Cristian GHEORGHE ${ }^{1,2}$, Adina ZAMFIR-CHIRU-ANTON ${ }^{3}$ \\ ${ }^{1}$ Department of Otorhinolaryngology, “M.S. Curie” Emergency Clinical Hospital for Children, \\ Bucharest \\ 2"Carol Davila" University of Medicine and Pharmacy, Bucharest \\ ${ }^{3}$ Department of Otorhinolaryngology, "Grigore Alexandrescu" Emergency Clinical Hospital for \\ Children, Bucharest
}

\begin{abstract}
Objective. To disscuss a less common complication of middle ear surgery. Meningoceles are dural herniations that can fill the mastoid cavities through congenital or aquired bony defects of the mastoid walls. They can complicate the postoperative course of surgery for chronic middle ear disease.

Material and method. Case presentation showing one patient who developed a mastoid meningocele after extensive cholesteatoma surgery.

Disscussion. The causes of dural herniation into the mastoid cavities are presented. Some hypothesis regarding the factors that favor the occurence of this complication are presented.

Conclusions. Surgical closing of the bone gap in the mastoid wall was the treatment we used. We can not ascertain yet the long term results of such surgical maneuver, regarding the technique and the graft we used. Attention to the mastoid surgical detail and good postoperative intensive care could prevent such complications.
\end{abstract}

Keywords: mastoidectomy, meningocele, cholesteatoma

Note. The authors contributed equally to this paper.

Mastoidectomy cavities are a well established means of dealing with chronic ear disease. Their utility has long been described. Their use, albeit lifesaving, bear sometimes an associated morbidity that makes the patient a continous medical services user. These cavities lose the normal capacity of the ear canal to clearance the cerumen secretions and can maintain a certain degree of local humidity, which in turn favors the chronic inflammation of the lining epithelium with posssible recurrent infection and cholesteatoma.

Some published papers also stress the fact that hearing threshoulds of a patient with mastoidectomy open cavity can be worse than ope- rated ears preserving the osseous canal wall up. That can be a matter of debate.

Other uncommon possible complications that can occur in connection with mastoidectomy cavity are meningoceles, through breaches of the bone that limits the tympano-mastoid cavities, during the initial surgery. Most of temporal bone meningoceles can be a consequence of the ear disease (9-21\%) (like cholesteatoma) or of the drilling process used to cure the chronic ear lesions (45-59\%). $(1,2)$ Their incidence is low, around 150 cases have been reported in the last 50 years. Other causes for temporal bone meningoceles include: malformations and trauma to the temporal bone. (2) 
The treatment options in these cases areall surgical, since an anatomic abnormality needs correction for a final and stable cure. Occlusion of the bone defect can be done using various materials: conchal cartilage (2), autologous cortical bone (3), hydroxyapatite cement. (4) The surgery can be performed through a mastoid or/and minitemporal craniotomy approach. (5) The combined approach is mentioned as a superior way of offering good exposure and minimal risks. (6)

In this paper, we present a cholesteatoma case patient, who developed a large meningocele in a mastoidectomy cavity, restant after cholesteatoma removal. The surgery that we performed is described and a long-term followup of the case is reviewed in order to better assess the possible evolutions for such condition.

\section{CASE PRESENTATION}

A 9 years old boy was admitted to our department for chronic left ear otorheea. He had been diagnosed with left ear cholesteatoma 1 year before. A radical mastoidectomy had been performed in another ENT surgical service. The left ear discharge did not stop after his surgery and intermitent headaches could be recorded during the past 3 months. A CT scan of the mastoid revealed a large meningocele developed through a bony gap of the superior part of the mastoid cavity.

Mastoid surgery was performed by posterosuperior approach, revealing an epithelial lined cavity, completely obstructed by thick dural her- niation, covered by granulation tissue, purulent discharge and diseminated foci of cholesteatoma in various left mastoid recesses. The inflammation made difficult the dissection of the dural hernia but we succeded a clear separation from the epidermal plane and elicited the bone defect of the mastoid tegmen. The communication between the mastoid and the cranial cavity was a $14 \mathrm{~mm}$ ovoid shaped bony defect, through which the dural tissue allowed to be pushed back to its normal location.

A round piece of bone, of about $15 \mathrm{~mm}$ diameter was harvested from the external cortex of the temporal bone. It was fixated on the inner side of the mastoid tegmen bony gap, after dural detachment from the bony margins and the hernia push into the cranial cavity. A rotation movement allowed the bone graft insertion with good fixation in the ovoid bony defect. The mastoid part of the bone gap was covered with aponeurosis and a large muscle fragment. The remnant cavity was cleared of remaining cholesteatoma and a tympanic membrane reconstruction was undertaken.

Postoperative course was uneventful, complete healing of the open mastoid cavity occuring 4 weeks after discharge of the patient.

\section{DISCUSSION}

Mastoidectomy cavities can be useful in mastoid surgery because they allow a thorough inspection of difficult areas and complete excision of middle ear disease. They are always the prefered surgical technique when intracranial
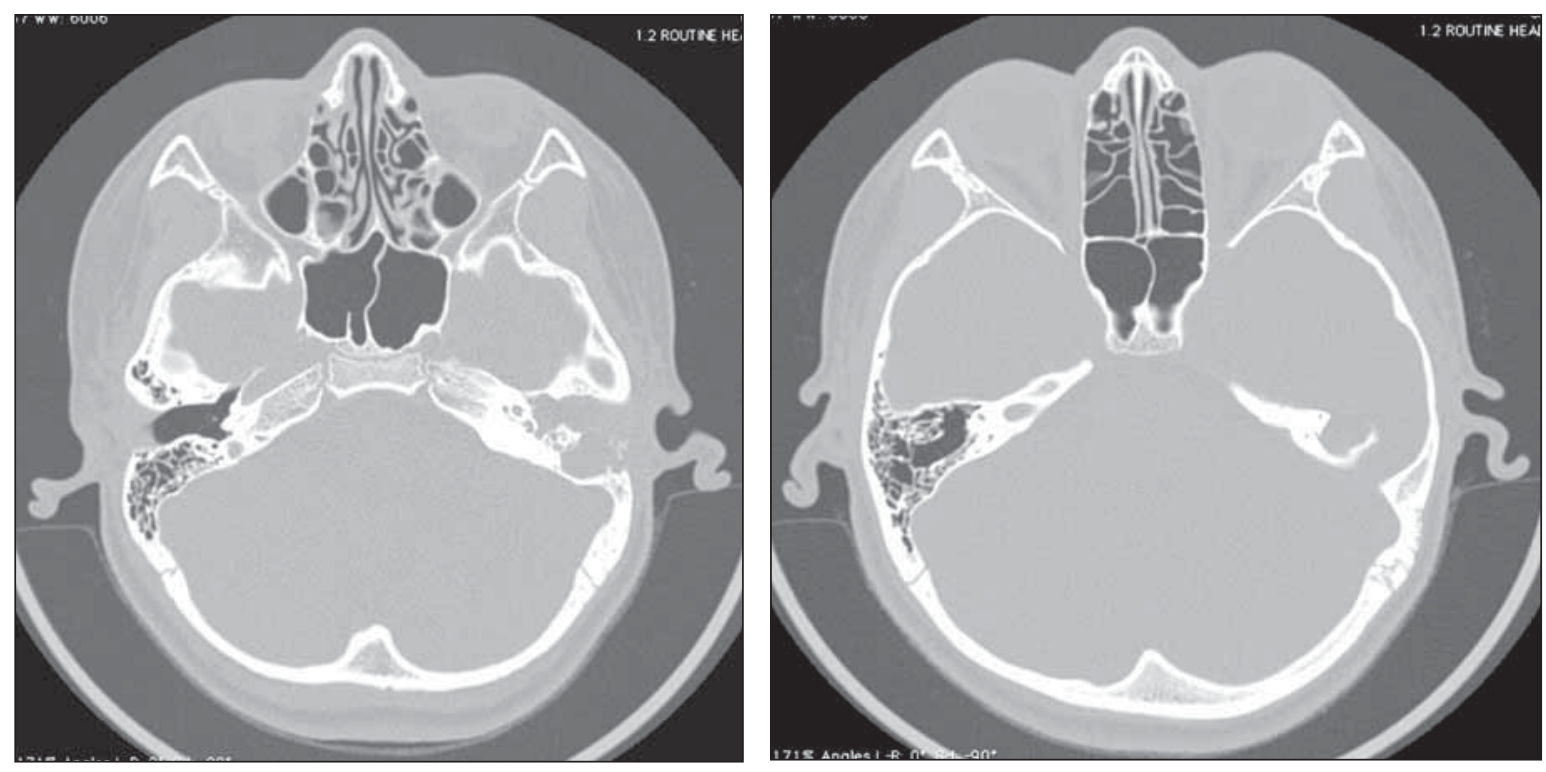

FIGURE 1. CT scan of the patient showing loss of bone from mastoid superior limit and cranial content entering mastoidectomy cavity 
complications exist. Other advantages relate to the ease with which they can be clinically monitored and the possibility to remove residual disease by simple surgical manoeuvers.

The down sides of mastoid cavities are the lack of a good epithelial and cerumen clearance and a high risk of cholesteatoma recurrence, due to inappropriate local ventilation/aeration. That needs periodic medical specialized care.

Mastoidectomy requires thorough drilling of the temporal bone, in order to completely remove the local disease (cholesteatoma, chronic osteitis). Sometimes, dural plate is uncovered by drilling towards the mastoid cavities. The bony defect can be either a consequence of a chronic ear disease (eg: cholesteatoma) or of excessive drilling (iatrogenic). Usually, the dura does not have the tendency to protrude into the mastoid cavity. There have been reports about the discrepancy between the incidence of temporal bone defects and the frequency of brain herniations, leading to the conclusion that not all bony defects can determine meningocele formation. (3) That is also commonly observed in mastoid surgery. Covering such bony defects with local tissues (aponeurosis) at the end of the surgical technique can add to the good cranial-mastoid isolation and add to prevention of possible recurrent meningitis.

Meningoceles can develop if large bone defects exist in the mastoid cavity walls. Mastoid surgery is the most common associated event in patients with temporal bone meningoceles developed after chronic ear disease. (6) The possible risks associated with such complication can be: recurrent meningitis (5), local chronic infection (otorheea) and cholesteatoma recurrence. Theoretically, the protruding dura can origin from both middle cranial fossa or posterior fossa. Still, gravitation can favor the former anatomic variant. Whether any associated events at surgery (intracranial edema, abscess, meningitis) do contribute to postoperative meningocele formation is a matter of debate. (7)

Clinical signs associated with meningocele formation in the presence of an operated ear were: mass into the mastoid cavity, chronic ear discharge, recurrent headaches. That physical picture is very similar to that reported by other authors. (8) Not all dural herniations are asociated with CSF leakage. (6)

The complication was confirmed by CT scan imaging. The meningo-encephalic tissue that proved contiguous with the mastoid mass helped our differential diagnosis.

Surgical treatment aimed at better separating the dura from the mastoid cavity lining, allowing its ventilation and healing and lowering the local chance of infection, with meningitis development risk. Neurosurgeons can be involved as well, craniotomy being a well described tool for meningocele approach. (6)

The patient presented in our paper was approached by the mastoid route. We used an autologous cortical temporal bone plate to fix the dural herniation tendency. The result was straitforward and was followed by a stable healing. Still, some questions remain unanswered. Can the bone graft survive a constant pressure from the dural part? Multilayered approach can add to stability. Does the grafting prevent meningeal infection in case of local mastoid disease? A long time monitoring can elucidate both issues. A similar method like ours is reported in the literature, with good results. (9)

The same method has been described in the literature as transmastoid extradural intracranial approach (TMEDIC) but using cartilage instead of cortical bone. $(10,11)$ There are also disscussions about a possible obliteration of the middle ear space (subtotal petrosectomy). $(2,12)$ However, in cases with good functional auditory reserve, that would be an extreme surgery with less than optimal results. Most of the authors dealing with this kind of lesion advocate brain tissue herniation removal. The reported risk of postoperative epilepsy (13) and infection after the re-inclusion of the hernia into the cranial cavity makes is not entirely sustained by others. (6)

\section{CONCLUSIONS}

Meningocele occurence after mastoidectomy is un uncommon complication, possibly seen in severe cholesteatoma cases that involves extensive drilling. Large defects in mastoid walls after surgery need multilayered grafting, in order to prevent dural herniation and associated infectious intracranial complications. A good intensive care after radical mastoidectomy cankeep a low intracranial pressure postoperatively and also prevent dural herniation.

Osseous grafting of the mastoid defects are relatively easy to perform. Their long-term reliability remains to be established. 


\section{REFERENCES}

1. Iurato S., Ettorre G.C., Selvini C. Brain herniation into the middle ear: two idiopathic cases treated by a combined intracranial-mastoid approach. Laryngoscope, 1989. 99(9): p. 950-4.

2. Sanna M., et al. Management of meningoencephalic herniation of the temporal bone: Personal experience and literature review. Laryngoscope, 2009. 119(8): p. 1579-85

3. Lundy L.B., et al. Temporal bone encephalocele and cerebrospinal fluid leaks. Am J Otol, 1996. 17(3): p. 461-9.

4. Gubbels S.P., et al. Spontaneous middle fossa encephalocele and cerebrospinal fluid leakage: diagnosis and management. Otol Neurotol, 2007. 28(8): p. 1131-9.

5. Feenstra L., et al. Surgical treatment of brain herniation into the middle ear and mastoid. Am J Otol, 1985. 6(4): p. 311-5.
6. Bodenez C., et al. Temporal breach management in chronic otitis media. Eur Arch Otorhinolaryngol, 2008. 265(11): p. 1301-8.

7. Mosnier l., et al. Brain herniation and chronic otitis media: diagnosis and surgical management. Clin Otolaryngol Allied Sci, 2000. 25(5): p. 385-91.

8. Sergi B., et al. Transmastoid approach to repair meningoencephalic herniation in the middle ear. Acta Otorhinolaryngol Ital, 2013. 33(2): p. 97-101.

9. Redaelli de Zinis L.O. Transmastoid repair of meningoencephalic herniation associated with cholesteatoma by canal wall-down procedure: our experience in eighteen patients. Clin Otolaryngol, 2013. 38(5): p. 397-402.

10. Manjila S., et al. Meningoencephalocele of the temporal bone: pictorial essay on transmastoid extradural-intracranial repair. Am J Otolaryngol, 2013. 34(6): p. 664-75.

11. Semaan M.T., et al. Transmastoid extradural-intracranial approach for repair of transtemporal meningoencephalocele: a review of 31 consecutive cases. Laryngoscope, 2011. 121(8): p. 1765-72.

12. Oliaei S., Mahboubi H., Djalilian H.R. Transmastoid approach to temporal bone cerebrospinal fluid leaks. Am J Otolaryngol, 2012. 33(5): p. 556-61.

13. Jackson C.G., et al. Brain herniation into the middle ear and mastoid: concepts in diagnosis and surgical management. Am J Otol, 1997. 18(2): p. 198-205; discussion 205-6.

Vizitați site-ul revistei

\section{Practica Medicală} pm.medica.ro 